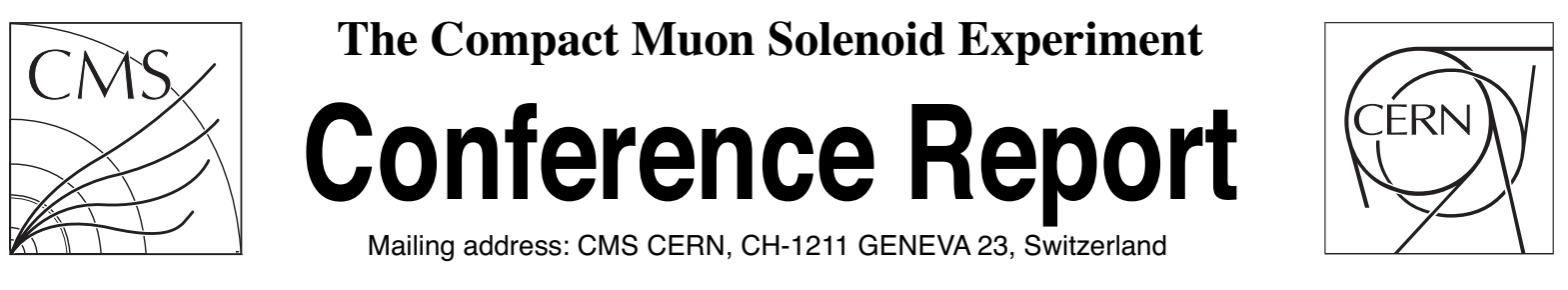

11 December 2019 (v3, 14 December 2019)

\title{
Study of the Effects of Radiation at the CERN Gamma Irradiation Facility on the CMS Drift Tubes Muon Detector for the HL-LHC
}

\author{
Archie Sharma for the CMS Muon Group
}

\begin{abstract}
The CMS drift tubes (DT) muon detector, built for withstanding the LHC expected integrated and instantaneous luminosities, will be used also in the High Luminosity LHC (HL-LHC) at a 5 times larger instantaneous luminosity and, consequently, much higher levels of radiation, reaching about 10 times the LHC integrated luminosity. Initial irradiation tests of a spare DT chamber at the CERN gamma irradiation facility $(\mathrm{GIF}++)$, at large $(\mathrm{O}(100))$ acceleration factor, showed aging effects resulting in a degradation of the DT cell performance however, full CMS simulations have shown almost no impact in the muon reconstruction efficiency over the full barrel acceptance and for the full integrated luminosity. A second spare DT chamber was moved inside the GIF++ bunker in October 2017. The chamber was being irradiated at lower acceleration factors, and only 2 out of the 12 layers of the chamber were switched on at working voltage when the radioactive source was active, being the other layers on standby. In this way the other non-aged layers were used as reference and as a precise and unbiased telescope of muon tracks for the efficiency computation of the aged layers of the chamber, when set at working voltage for measurements. An integrated dose equivalent to two times the expected integrated luminosity of the HL-LHC run had been absorbed by this second spare DT chamber and the final impact on the muon reconstruction efficiency was measured. Direct inspection of some extracted aged anode wires presented a deposition of materials. Investigation on the outgassing of cell materials and of the gas components used at the GIF++ are underway. Strategies to mitigate the aging effects are also being developed. From the long irradiation measurements of the second spare DT chamber, the effects of radiation in the performance of the DTs expected during the HL-LHC run will be presented.References [1] CMS-TDR-016, The Phase-2 Upgrade of the CMS Muon Detectors
\end{abstract}

Presented at IEEE-NSSMIC2019 2019 IEEE Nuclear Science Symposium (NSS) and Medical Imaging Conference (MIC) 


\section{Study of the Effects of Radiation at the CERN Gamma Irradiation Facility on the CMS Drift Tube Muon Detector for HL-LHC}

G. Abbiendi ${ }^{a}$, J. Alcaraz Maestre ${ }^{b}$, A. Álvarez Fernández ${ }^{b}$, B. Álvarez González ${ }^{c}$, N. Amapane ${ }^{d, e}$, I. Bachiller $^{b}$,

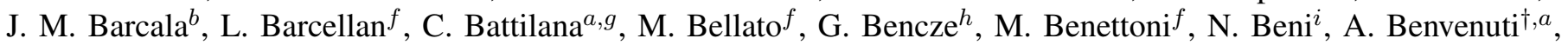
L. C. Blanco Ramos ${ }^{b}$, A. Boletti ${ }^{f}$, A. Bragagnolo ${ }^{f}$, J. A. Brochero Cifuentes ${ }^{b}$, V. Cafaro ${ }^{a}$, A. Calderon ${ }^{j}$, E. Calvo ${ }^{b}$, A. Cappati ${ }^{d, e}$, R. Carlin ${ }^{f}$, C. A. Carrillo Montoya ${ }^{b}$, F. R. Cavallo ${ }^{a}$, J. M. Cela Ruiz ${ }^{b}$, M. Cepeda ${ }^{b}$, M. Cerrada ${ }^{b}$, B. Chazin Quero $^{j}$, P. Checchia ${ }^{f}$, L. Ciano ${ }^{f}$, N. Colino ${ }^{b}$, D. Corti ${ }^{f}$, G. Cotto ${ }^{d, e}$, J. Cuevas ${ }^{c}$, M. Cuffiani ${ }^{a, g}$, G. M. Dallavalle ${ }^{a}$, D. Dattola ${ }^{d}$, B. De La Cruz ${ }^{b}$, P. De Remigis ${ }^{d}$, J. F. de Trocóniz ${ }^{k}$, C. Erice Cid $^{c}$, C. F. Bedoya ${ }^{b}$, F. Fabbri ${ }^{a}$, A. Fanfani ${ }^{a, g}$, D. Fasanella ${ }^{a, g, p}$, P. Fernandez Manteca ${ }^{j}$, J. Fernández Menéndez $^{c}$, J. P. Fernández $\operatorname{Ramos}^{b}$, S. Folgueras ${ }^{c}$, M. C. Fouz ${ }^{b}$, D. Francia Ferrero ${ }^{b}$, J. García Romero ${ }^{b}$, F. Gasparini ${ }^{f}$, U. Gasparini ${ }^{f}$, V. Giordano ${ }^{a}$, F. Gomez Casademunt ${ }^{j}$, F. Gonella ${ }^{f}$, I. González Caballero ${ }^{c}$, J. R. González

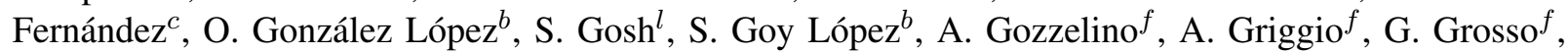
C. Guandalini ${ }^{a}$, L. Guiducci ${ }^{a, g}$, M. Gulmini ${ }^{e, m}$, T. Hebbeker ${ }^{l}$, C. Heidemann ${ }^{l}$, J. M. Hernández ${ }^{b}$, K. Hoepfner ${ }^{l}$,


J. Marín ${ }^{b}$, C. Mariotti ${ }^{d}$, I. Martín Martín ${ }^{b}$, J. J. Martínez Morales ${ }^{b}$, C. Martínez Rivero ${ }^{j}$, S. Maselli ${ }^{d}$, G. Masetti ${ }^{a}$, A. T. Meneguzzo ${ }^{f}$, M. Merschmeyer ${ }^{l}$, G. Mocellin ${ }^{f, q}$, L. Modenese $^{f}$, A. Molinero ${ }^{b}$, J. Molnar $^{i}$,

F. Montecassiano ${ }^{f}$, D. Moran ${ }^{b}$, J. J. Navarrete ${ }^{b}$, F. Navarria ${ }^{a, g}$, Á. Navarro Tobar ${ }^{b}$, J. C. Oller ${ }^{b}$, M. Passaseo ${ }^{f}$, J. Pazzini ${ }^{f}$, M. Pegoraro ${ }^{f}$, J. Puerta Pelayo ${ }^{b}$, M. Pelliccioni ${ }^{d}$, B. Philipps ${ }^{l}$, J. Piedra Gomez ${ }^{j}$, G. L. Pinna



D. D. Redondo Ferrero ${ }^{b}$, H. Reithler ${ }^{l}$, T. Rodrigo ${ }^{j}$, V. Rodríguez Bouza ${ }^{c}$, J. Roemer $^{l}$, P. Ronchese ${ }^{f}$, R. Rossin $^{f}$,

F. Rotondo ${ }^{d}$, T. Rovelli ${ }^{a, g}$, S. Sánchez Cruz ${ }^{c}$, S. Sánchez Navas ${ }^{b}$, J. Sastre $^{b}$, L. Scodellaro ${ }^{j}$, A. Sharma ${ }^{l}$,

F. Simonetto ${ }^{f}$, M. S. Soares ${ }^{b}$, A. Staiano ${ }^{d}$, Z. Szillasi ${ }^{i}$, D. F. Teyssier ${ }^{i}$, N. Toniolo ${ }^{e, m}$, E. Torassa $^{f}$, D. Trocino ${ }^{d}$, B. $\operatorname{Ujvari}^{n}$, S. Ventura ${ }^{f}$, R. Vilar Cortabitarte ${ }^{j}$, J. Vizan Garcia $^{j}$, M. Zanetti ${ }^{f}$, F. P. Zantis ${ }^{l}$, G. Zilizi ${ }^{n}$, P. Zotto ${ }^{f}$, on behalf of the CMS Muon group

${ }^{a}$ INFN Sezione di Bologna, Italy

${ }^{b}$ Centro de Investigaciones Energéticas Medioambientales y Tecnológicas (CIEMAT), Spain

${ }^{c}$ Instituto Universitario de Ciencias y Tecnologías Espaciales de Asturias (ICTEA), Universidad de Oviedo, Spain ${ }^{d}$ INFN Sezione di Torino, Italy

${ }^{e}$ Universit di Torino, Italy

${ }^{f}$ INFN Sezione di Padova; Universit di Padova, Italy

${ }^{g}$ Universit di Bologna, Italy

${ }^{h}$ Wigner Research Centre for Physics, Hungary

${ }^{i}$ Institute of Nuclear Research ATOMKI, Hungary

${ }^{j}$ Instituto de Fsica de Cantabria (IFCA), CSIC-Universidad de Cantabria, Spain

${ }^{k}$ Universidad Autnoma de Madrid (UAM), Spain

${ }^{l}$ RWTH Aachen University, III. Physikalisches Institut A, Aachen, Germany

${ }^{m}$ Laboratori Nazionali di Legnaro dell'INFN, Italy

${ }^{n}$ Institute of Physics, University of Debrecen, Hungary

${ }^{o}$ Italian National Agency for New Technologies, Energy and sustainable economic development, Bologna, Italy

${ }^{p}$ Now at CERN

${ }^{q}$ Now at RWTH Aachen University

${ }^{\dagger}$ Deceased

The corresponding author of this manuscript A. Sharma is with
III. Physikalisches Institut A, RWTH Aachen University (e-mail: archie.sharma@cern.ch)
Abstract-The CMS drift tubes (DT) muon detector, built for withstanding the LHC expected integrated and instantaneous luminosities, will be used also in the High Luminosity LHC 
(HL-LHC) at a 5 times larger instantaneous luminosity and, consequently, much higher level of radiation, reaching about 10 times the LHC integrated luminosity. Initial radiation tests of a spare DT chamber at the CERN gamma irradiation facility (GIF++), at large $(\sim \mathcal{O}(100))$ acceleration factor, showed aging effects resulting in a degradation of the DT cell performance; however, full CMS simulations have shown almost no impact in the muon reconstruction efficiency over the full barrel acceptance and for the full integrated luminosity. A second spare DT chamber was moved inside the GIF++ bunker in October 2017. The chamber was being irradiated at lower acceleration factors, and only 2 out of 12 layers of the chamber were switched on at working voltage when the radioactive source was active, being the other layers on standby. In this way the other non-aged layers were used as reference and as a precise and unbiased telescope of muon tracks for the efficiency computation of the aged layers of the chamber, when set at working voltage for measurements. An integrated dose equivalent to two times the expected integrated luminosity of the HL-LHC run had been absorbed by this second spare DT chamber and the final impact on muon reconstruction efficiency was measured. Direct inspection of some extracted aged anode wires presented a deposition of materials. Investigation on the outgassing of cell materials and of the gas components used at the GIF++ are underway. Strategies to mitigate the aging effects are also being developed. From the long irradiation measurements of the second spare DT chamber, the effects of radiation in the performance of the DTs expected during the HL-LHC run are presented.

\section{INTRODUCTION}

$\mathbf{T}$ O sustain and extend its discovery potential, the Large Hadron Collider (LHC) will undergo a major upgrade in the coming years referred to as High Luminosity LHC [1] aimed to increase its instantaneous and integrated luminosity respectively by a factor of five and ten beyond the original design value. In order to meet the experimental challenges of unprecedented proton-proton luminosity, the experiments will need to address the aging of the present detectors and to improve the ability to isolate and precisely measure the products of the most interesting collisions. The Compact Muon Solenoid (CMS) detector [2] also aims to upgrade its electronics and detector performance to improve the data taking and precise reconstruction of all the particles in the high-pileup conditions of the HL-LHC. The Drift Tubes subdetector is an essential part of the CMS muon system and plays a key role in the identifying and measuring and triggering on muons. In order to estimate the effects due to the higher instantaneous and integrated dose expected during the HL-LHC in the DT chambers [3], an irradiation campaign has been conducted in the CERN Gamma Irradiation Facility (GIF++) [4].

\section{A. The CMS Drift Tube subdetector (DT)}

The CMS DT system [5] is the main responsible of correctly identifying muons in the CMS barrel region, consists of 250 chambers located in 5 wheels (Wh-2, Wh- $1, \mathrm{Wh} 0, \mathrm{Wh}+1$, $\mathrm{Wh}+2$ ) inside $|\eta|<1.2$. Each wheel is subdivided in 12 sectors, covering different $\phi$ regions. Each sector is composed by four DT stations (or chambers) (except for horizontal sectors, 4 and 10 , that contain 5 stations). The innermost stations of each sector are called MB1 stations and the stations next to the radial direction as MB2, MB3 and MB4 respectively. The layout of

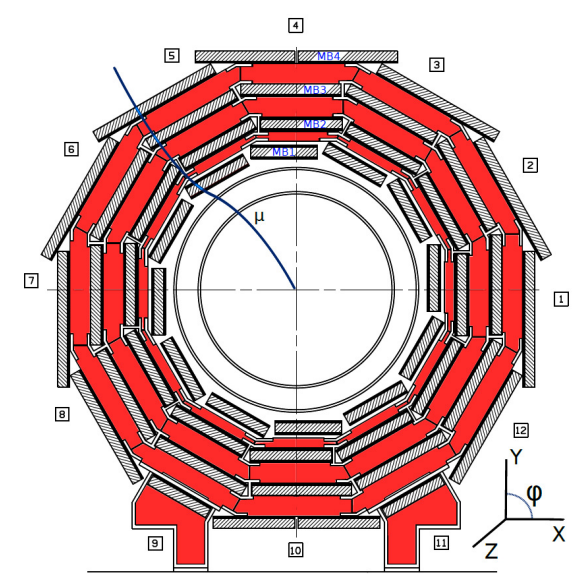

Fig. 1. Transverse view of a CMS barrel wheel of the DT system.

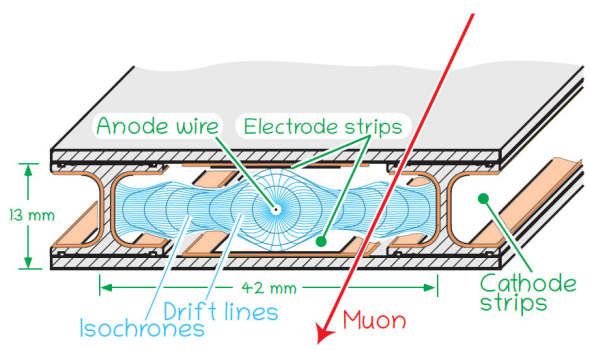

Fig. 2. Layout of a CMS DT cell.

one such DT wheel is shown in Fig. 1. Each DT chamber is a glued structure of about 700 cells arranged in 12 layers (or 8 for the MB4 stations). The layers are grouped in packs of 4 (represented as L1, L2, L3 and L4) called superlayers (SL) and depending on the orientation of the cells, the SL are called $\Theta$ if the cells are oriented in $\mathrm{Z}$ direction and $\Phi$ if the cells are along the $\phi$ direction. A DT cell consists of a central anode wire of $50 \mu \mathrm{m}$ in diameter with two cathode strips at each side of the cell and a pair of positively-charged strips situated in the upper and lower parts of the cell as shown in Fig. 2. The cells are filled with a gas mixture of $85 \% \mathrm{Ar}$ and $15 \% \mathrm{CO}_{2}$. The passage of muons through the DT cells causes ionization in the atoms of the gas and in the standard working conditions of DTs, a gas gain of about $10^{5}$ is reached. The collected charge in the wire is read by the front-end (FE) electronics, amplified at the FE board and compared with a nominal value of threshold (FEth). Further description of this system can be found in [5].

\section{B. The Gamma Irradiation Facility $(G I F++)$}

GIF++ is a unique facility where high energy charged particle beams (mainly muons) from the SPS are combined with a flux of photons from a $13.9 \mathrm{TBq}{ }^{137} \mathrm{Cs}$ source which is situated inside the bunker with several gaseous detectors in order to test them under different radiation conditions. A filter system permits attenuating the photon rate independently in the two areas inside the bunker (upstream and downstream corresponding to the side of the source closer to or further from the beam starting point). The high intensity of the GIF++ 
source allows to perform accelerated irradiation tests and the filters are used to regulate the intensity of the source, not to surpass more than ten times the dose rate expected at HLLHC for the most exposed DT chambers of the CMS detector. The muon beam allows the characterization of the detection efficiency with and without background irradiation provided by the source. Scintillators provided trigger on the beam muons, while the chamber has also auto-trigger capability, which was used for cosmic muon data taking during periods without beam.

\section{EXPERIMENTAL SETUP}

A spare DT MB2 chamber was introduced in the GIF++ bunker in September 2017 and irradiated in two campaigns that finished in January 2019. The chamber was situated at about $4 \mathrm{~m}$ from the source, in the downstream part of GIF++ bunker, close to the wall. The chamber was standing in vertical position, with the SL1 facing the source. A huge amount of data were collected during the irradiation, including a constant monitoring of all the parameters, weekly measurements with cosmic muons. Data with a muon beam was also recorded in October 2017 and July 2018. In the experiment, two layers (L1 and L4) in SL1 were used to test the aging effect with their anode $\mathrm{HV}$ at working voltage $(3550 \mathrm{~V}$ and $1900 \mathrm{~V})$ when the radioactive source was switched on. Other layers (L2 and L3) in the SL1 were kept on standby $(1900 \mathrm{~V})$ and were used as reference layers, along with the rest of chamber (SL2 and SL3) which was also on standby so the gas gain is negligible. This arrangement preserved the other layers and so they were used as a precise and unbiased telescope for muon tracks.

\section{A. Dose measurement and conversion factors}

At GIF++, the amount of charge absorbed by the detector is proportional to the radiation dose caused by $662 \mathrm{KeV}(50 \%)$ and low energy photons from ${ }^{137} \mathrm{Cs}$ source, which causes the aging of the detector in the long term. A permanent REMUS dosimeter [6] in the GIF++ bunker monitors the dose rate. The photon dose rate of the ${ }^{137} \mathrm{Cs}$ source collected by REMUS depends on the attenuation filters that are set at any moment and the amount of materials situated between source and this dosimeter. For this reason, detailed measurements with a Geiger-Müller tube portable dosimeter (Automess 6150 AD) were used to extrapolate dose rates from the REMUS position to the real location of the chamber. The total accumulated dose were computed at any time by integrating the dose rate over the periods when the aged layers were kept on. At CMS, the integrated dose received by any of the DT stations is proportional to the integrated luminosity along the time. A conversion factor from integrated dose at GIF++ to the expected integrated luminosity at CMS was derived for the most exposed chamber (MB1 chambers in the external wheels (Wh \pm 2 ) of the CMS detector): $1 \mathrm{fb}^{-1}=0.42 \mathrm{mGy}$. Furthermore, the background rate also affects the performance of the detector. The muon hit efficiency is degraded by the presence of a background of particles that can affect the signal collected by the anode wires or introduce background noise to the measurements. The effect of the background rate at GIF++ is proportional to the photon dose rate from the source,

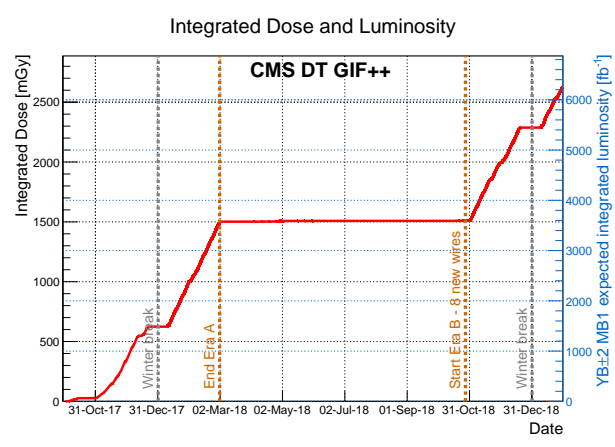

Fig. 3. Integrated dose (mGy) as a function of time over the irradiated layers (L1 and L4) in SL1. The data taking is divided into two periods: the first, called era A, started in October 2017 and ended in April 2018, after irradiating the chamber with amount of radiation equivalent to that expected for the full HLLHC run. After that period, the DT chamber was open and a few wires were extracted for inspection. The era B started at the end of October 2018 and continued until end of January 2019.

while for CMS DT system, the amount of background rate for each DT station depends on the instantaneous luminosity given by the LHC. The relation between these two quantities at GIF++ and CMS DT system is $10^{34} \mathrm{~cm}^{-2} \mathrm{~s}^{-1}=0.0109$ $\mathrm{mGyh}^{-1}$. Fig. 3 shows the integrated dose collected by the MB2 chamber at GIF++ and its equivalent HL-LHC expected integrated luminosity for a MB1 chamber of the CMS DT system in wheels \pm 2 .

\section{B. Hit efficiency}

The goal of the CMS muon detector is to provide identification, track reconstruction and trigger of muons and the DT hit detection efficiency plays a key role to achieve this. A big effort was put in characterizing this quantity with aging of the cells. The efficiency to detect a single hit in a cell of a layer was defined and measured as the ratio between the number of selected and expected hits. The position of expected hits was determined using as probes well reconstructed track segments with associated hits in at least 4 layers in SL3 and at least 1 layer in SL1. The intersection of this track segment with the layer under study determined the position, therefore the cell, where a hit was expected; the cell was considered efficient if a hit was found within it. A layout of the measurements of the hit efficiency is shown in Fig. 4. The hit efficiency was measured periodically during the irradiation of the chamber, under different working conditions, in the aged layers, while the non-aged layers in SL1 (L2 and L3) were used as reference.

\section{RESULTS}

After the end of the irradiation period, a total accumulated dose equivalent to about twice the expected integrated luminosity at the end of the HL-LHC for the most irradiated DT chambers had been reached. For most of the data taking, cosmic muons were used to measure the efficiency at a $\mathrm{HV}$ value of $3550 \mathrm{~V}$ for the two aged layers, with source off and FEth $=30 \mathrm{mV}$. Cosmic muons were recorded using the DT auto trigger requiring a track in both projections on just SL2 and SL3 of the DT chamber. Evolution of the hit efficiency in the 


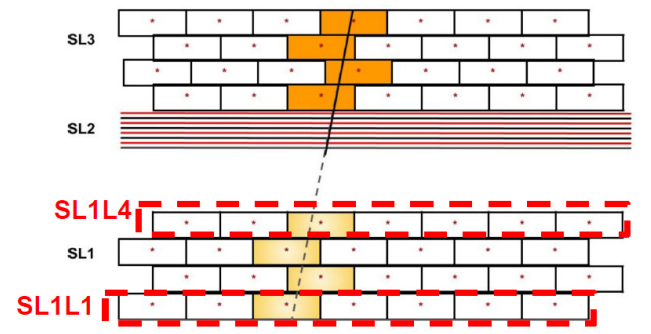

Fig. 4. Layout of the measurement of the hit efficiency at GIF++ for any layer in SL1. The SL3 is used as reference and the reconstructed segment is extrapolated to the test layer in SL1.

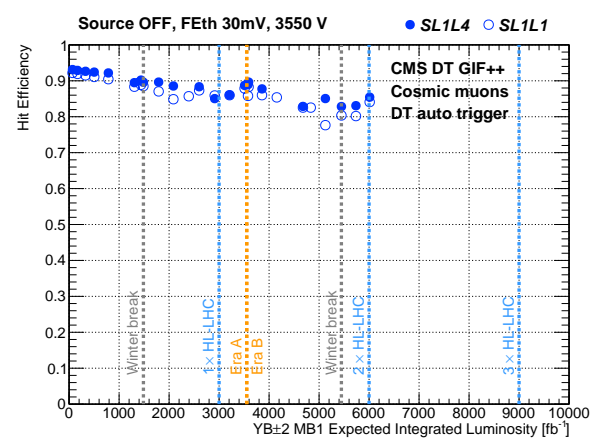

Fig. 5. Evolution of the hit efficiency with the accumulated radiation for layers 1 and 4 of SL1. The data were taken almost each week during the irradiation period using cosmic muons, with a FEth of $30 \mathrm{mV}$ and source off. The accumulated radiation is expressed in terms of the equivalent expected integrated luminosity for a MB1 DT chamber at CMS Wh \pm 2 during $\mathrm{HL}$ LHC.

aged layer using cosmic muons as a function of the equivalent integrated luminosity for the most exposed chambers is shown in Fig. 5. A moderate efficiency loss is observed in both aged layers of about $10 \%$ for an accumulated irradiation equivalent to twice the expected integrated luminosity at the HL-LHC.

To have a more realistic scenario, data were taken with high momentum beam muons together with the source in place using different filters to see the dependency of the hit efficiency on background rate. GIF++ external scintillators were used to trigger these muons. Fig. 6 shows the dependence of the hit efficiency with the instantaneous luminosity for the aged layers when the chamber had accumulated a dose of bit larger than that expected at the HL-LHC. The hit efficiency degraded about $20 \%$ in the presence of background radiation. The non irradiated layers show no drop.

All the measurements summarize the characterization of the expected aging of a DT chamber at the HL-LHC. In particular, this study was done to see the effect of the aging in terms of expected instantaneous and integrated luminosity at the HLLHC for a MB1 chamber at wheels \pm 2 , which will be the most irradiated DT chambers. To extrapolate the measured efficiency to the rest of the DT chambers of the CMS muon system, the expected background rate and integrated luminosity were obtained from the measurements of the integrated charge during 2018 data taking at CMS for the 250 DT stations, correcting by the expected changes in the detector before the starting of the HL-LHC run. The efficiencies were calculated

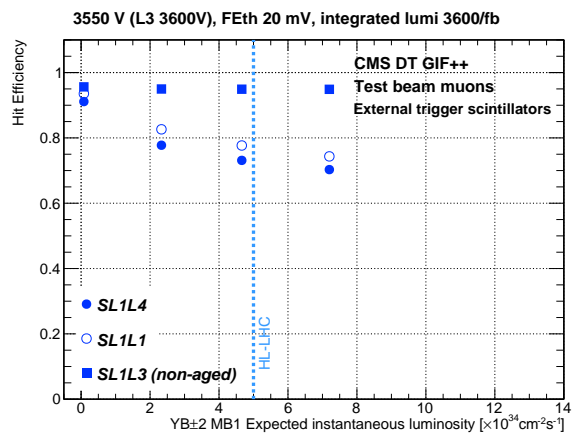

Fig. 6. Hit efficiency for the test beam muons as a function of instantaneous luminosity for aged layers (SL1L1 and SL1L4) and a non-aged layer (SL1L3). Data taken after an accumulated dose equivalent to an HL-LHC integrated luminosity of $3600 \mathrm{fb}^{-1}$.
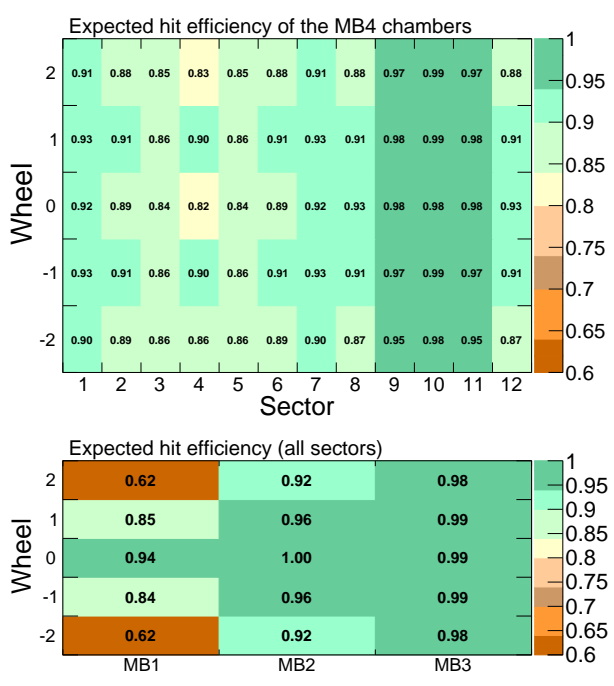

Fig. 7. Estimated hit efficiencies at the end of the HL-LHC for all the DT chambers of the CMS muon system, MB4 chambers (upper) and for MB1, MB2 and MB3 (lower). These efficiencies have been estimated considering a safety factor of 2 for the expected HL-LHC background rate $\left(10^{35} \mathrm{~cm}^{-2} \mathrm{~s}^{-1}\right)$ and integrated luminosity $\left(6000 \mathrm{fb}^{-1}\right)$ to obtain the expected hit efficiency for the MB1 chambers in wheel \pm 2 and extrapolating to the rest of the CMS muon system using the expected integrated charge at the end of HL-LHC.

per DT station, assuming that they are equal for all the layers and the cells in each station. A safety factor of 2 was considered in both the expected integrated luminosity and the background rate to cope with the various uncertainties in the final HL-LHC conditions and the data taking. The values obtained are shown in Fig. 7.

To study the effect of aging on the overall CMS muon reconstruction, the muon reconstruction efficiency were calculated from a simulated sample generated using the hit efficiencies obtained for each DT chamber in Fig. 7. Fig. 8 shows the muon reconstruction efficiency as a function of the pseudorapidity $(\eta)$ of the muon and compared to an ideal scenario of non-aged DT chambers. The variation of the efficiency is expected to be negligible over almost the whole $\eta$ range. 


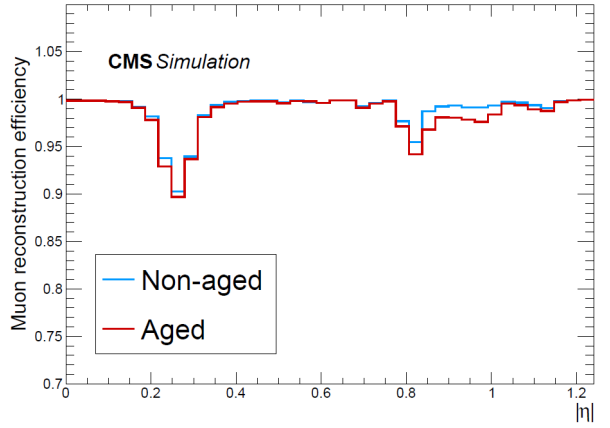

Fig. 8. Muon reconstruction efficiency at CMS as a function of $|\eta|$ for non aged DT chambers and aged DT chambers with the efficiencies shown in Fig. 7. The efficiencies have been calculated using simulated sample of dimuon events.

\section{CONCLUSIONS}

Drift cells are known to lose performance as they accumulate radiation. In the harsher environment of HL-LHC, where the radiation hitting the detector will be excessively high, the irradiation campaigns have provided critical information about the behavior of the detector in these conditions. The additional background rate coming with the increase of instantaneous luminosity has the capability of further affecting the performance of the aged detectors. The data collected by irradiating a spare DT chamber at GIF++ has provided very valuable data to characterize the response of these chambers at CMS detector throughout the HL-LHC operation. The hit efficiency drop for the MB1 chambers in wheel \pm 2 , that will be the most irradiated DT chambers at CMS, is estimated to be of the order of $38 \%$ at $6000 \mathrm{fb}^{-1}$ which is twice the expected integrated luminosity by the end of HL-LHC. The rest of the DT system shows smaller efficiency variations due to the lower background exposure and therefore lesser accumulated dose. However this drop in hit efficiency has a very small effect on the overall CMS muon reconstruction efficiency. Furthermore, the characterization of the expected hit efficiency drop at the HL-LHC will be used to estimate the effect of the aging on the performance of trigger efficiency. The evaluation of this effect will be crucial to design and improve trigger algorithms. Mitigation strategies have been implemented already to reduce the possibility of aging. Since the majority of the radiation that affects the chambers comes from the scattering on the walls and the activated material in the CMS experimental cavern, a $7 \mathrm{~mm}$ lead and $30-90 \mathrm{~cm} \mathrm{5 \%}$ borated polyethylene shielding is being installed on top of the most exposed DT chambers. At the same time the operational HV of the chambers has been reduced keeping the same performance of the detector by reducing the FEth from $30 \mathrm{mV}$ to $20 \mathrm{mV}$. Finally, investigation of the effect of additional components to current gas mixtures that may eventually moderate the coating deposition is planned.

\section{REFERENCES}

[1] G. Apollinari, I. Béjar Alonso, O. Brüning, M. Lamont, L. Rossi, HighLuminosity Large Hadron Collider (HL-LHC), Preliminary Design Report, 2015.
[2] CMS Collaboration, The CMS experiment at the CERN LHC, JINST 3 (2008).

[3] CMS Collaboration, The phase-2 upgrade of the CMS muon detectors, CERN-LHCC-2017-012, CMS-TDR, CERN(2017).

[4] R. Guida, The new CERN Irradiation Facility to test large-area detectors for $\mathrm{LHC}, 2016$.

[5] CMS Collaboration, The CMS muon project: Technical Design Report, CERN-LHCC-97-032, CERN(1997).

[6] A. Ledeul, G. Segura, R. Silvola, B. Styczen, D. V. Ribeira, REMUS: The new CERN Radiation and Environment Monitoring Unified Supervision, 2015. 